\title{
Aberrant Meiotic Configurations Cause Sterility in Clone-Origin Triploid and Inter-Group Hybrid Males of the Dojo Loach, Misgurnus anguillicaudatus
}

\author{
Masamichi Kuroda ${ }^{\text {a, }}$ Takafumi Fujimoto ${ }^{a}$ Masaru Murakami ${ }^{b}$ \\ Etsuro Yamahac Katsutoshi Arai ${ }^{\mathrm{a}}$ \\ ${ }^{a}$ Faculty of Fisheries Sciences, Hokkaido University, Hakodate, Hokkaido, Japan; 'b School of Veterinary Medicine, \\ Azabu University, Sagamihara, Kanagawa, Japan; ${ }^{C}$ Nanae Freshwater Station, Field Science Center for Northern \\ Biosphere, Hokkaido University, Nanae, Hokkaido, Japan; `Japan Society for the Promotion of Science, Tokyo, Japan
}

\section{Keywords}

Asynapsis · FISH · Hybrid · Meiosis · Sterility

\begin{abstract}
Gonochoristic wild-type dojo loaches (Misgurnus anguillicaudatus) are diploid $(2 n=50)$ and reproduce bisexually. However, sympatric clonal diploids generate unreduced diploid isogenic eggs that develop gynogenetically. Cloneorigin triploidy arises following the incorporation of a haploid wild-type sperm nucleus into the diploid egg. Triploid females produce fertile haploid eggs by meiotic hybridogenesis, while triploid males are sterile. Clonal loaches arose from past hybridization event(s) between genetically diverse groups, A and B. Artificial hybrid females between the 2 groups produce unreduced and/or aneuploid eggs, but the hybrid males are sterile. In this study using FISH, we analyzed chromosome pairing in meiotic cells of clone-origin triploid and inter-group hybrid males to clarify the cytogenetic mechanisms underlying the male-specific sterility. We used a repetitive sequence probe to identify group B-derived chromosomes and a $5.8 \mathrm{~S}+28 \mathrm{~S}$ rDNA probe to identify pairs of homologous chromosomes. We found that asynap-
\end{abstract}

sis and irregular synapsis occur in triploid and hybrid males containing 2 different genomes and that this may cause the formation of sterile germ cells. These results will help us to understand hybrid sterility from the viewpoint of synapsis behavior.

(c) 2019 S. Karger AG, Basel

Diploid wild-type dojo loaches, Misgurnus anguillicaudatus (Cobitidae, Cypriniformes, Teleostei), are gonochoristic and reproduce bisexually in most Japanese populations [Arai and Fujimoto, 2013]. However, clonal individuals appear in several populations in Hokkaido and Ishikawa Prefectures, Japan [Morishima et al., 2002, 2008a]. Clonal dojo loaches are essentially all female, because this species has a male heterogametic (XX female, XY male) sex determination system [Suzuki et al., 1985], and thus no contribution of the $\mathrm{Y}$ chromosome is predicted in clonal progeny. The clonal females generate unreduced isogenic diploid eggs, which develop by gynogenesis triggered by sperm from sympatric wild-type diploid males [Morishima et al., 2002; Itono et al., 2006, 2007]. Our genetic studies revealed that clonal loaches

\section{KARGER}

(c) 2019 S. Karger AG, Basel

E-Mail karger@karger.com

www.karger.com/cgr
Masamichi Kuroda

Faculty of Fisheries Sciences

Hokkaido University

Hakodate, Hokkaido 041-8611 (Japan)

E-Mail shindou19900120@eis.hokudai.ac.jp 
were heterozygotes that originated from past hybridization event(s) presumably between genetically diverse group A females and group B males [Khan and Arai, 2000; Arias-Rodriguez et al., 2007; Morishima et al., 2008a; Yamada et al., 2015] as previously reported in other unisexual vertebrates [Dawley, 1989; Vrijenhoek, 1994; Lutes et al., 2010]. Recently, nuclear DNA markers based on repetitive sequences were developed to distinguish the genomes of group A and B loaches [Fujimoto et al., 2017]. One of these, ManDra, exclusively detects 25 group B loach chromosomes (haploid number) in the diploid metaphases of clonal loaches with a $2 \mathrm{n}=50$ karyotype by FISH [Kuroda et al., 2018]. Both in meiosis of oocytes from clonal females and spermatocytes from artificially sex-reversed clonal males, 50 bivalents were easily counted. Thus, pairing should occur between sister chromosomes doubled from each ancestral chromosome from 2 different groups by a mechanism of premeiotic endomitosis [Itono et al., 2006; Yoshikawa et al., 2007, 2009; Kuroda et al., 2018].

Therefore, clonal loaches are of hybrid origin with a heterozygous genotype $(\mathrm{AB})$, including group $\mathrm{A}$ and $\mathrm{B}$ chromosome sets, and clonally reproduce by gynogenesis. However, isogenic diploid eggs sometimes incorporate the haploid sperm nucleus of sympatric wild-type diploids to become clone-origin triploids with 2 chromosome sets (genomes) of group A and 1 chromosome set of group B, i.e., trigenomic type AAB [Itono et al., 2007; Morishima et al., 2008b]. Such triploid females produce haploid eggs including chromosomes exclusively from group A by quasi-normal meiosis after eliminating unmatched group B chromosomes through meiotic hybridogenesis during oogenesis [Morishima et al., 2008b]. In contrast, triploid males produce few haploid, triploid, and hexaploid spermatozoa or spermatozoon-like cells, most of which have no motility after the addition of ambient water and exhibit morphological abnormalities such as larger head sizes and short, or no, flagellum [Oshima et al., 2005]. None or very few progeny appear after the fertilization of normal eggs with sperm of cloneorigin triploid males, indicating that clone-origin triploid males are post-zygotic sterile [Oshima et al., 2005].

While the clonal loach is of hybrid origin, artificial hybrids between extant genetic groups A and B have different reproductive consequences. Although such hybrid females produce fertile diploid and/or aneuploid eggs, the resultant unreduced diploid eggs never develop gynogenetically and become triploid progeny by incorporating a sperm nucleus at the time of fertilization [Arias-Rodriguez et al., 2009]. In contrast, hybrid males produce hap-

Hybrid Fish Sterility Caused by Aberrant Meiotic Configurations loid, diploid, and tetraploid cell populations in testis and semen, most of which exhibit an abnormal morphology [Arias-Rodriguez et al., 2010]. Very few diploid and triploid progeny appear after fertilization of normal eggs with such sperm, suggesting that inter-group hybrid males are sterile [Arias-Rodriguez et al., 2010].

Both females and artificially sex-reversed males of clonal diploid loaches are fertile [Morishima et al., 2002; Itono et al., 2006, 2007; Yoshikawa et al., 2007, 2009], but clone-origin triploids and inter-group hybrids males are sterile [Oshima et al., 2005; Arias-Rodriguez et al., 2010]. All of these biotypes are essentially of hybrid origin and include chromosome sets (genomes) from the genetically different groups A and B [Khan and Arai, 2000; AriasRodriguez et al., 2007; Morishima et al., 2008a, b; Yamada et al., 2015; Kuroda et al., 2018]. The genomic constitution of clonal diploid, clone-origin triploid, and artificial hybrids can be designated as $\mathrm{AB}, \mathrm{AAB}$, and $\mathrm{AB}$, respectively. Why clone-origin triploids and artificial hybrids exhibit male sterility and clonal diploids of both sexes are fertile, in spite of their similar heterozygous genomic constitutions, remains unknown. There are relatively few cytogenetic studies on the mechanisms of hybrid sterility in fish and other vertebrates, even though there are numerous reports of hybrid formation [Schwenk et al., 2008]. Unbalanced meiotic configurations, including univalent and multivalent chromosomes, are frequently observed in interspecific hybrids of mammals [Bhattacharyya et al., 2013; Torgasheva et al., 2016], birds [Islam et al., 2013], amphibians [Müller, 1977], and fish [Shimizu et al., 1997], indicating failure of synapsis as the cause of postzygotic sterility. To the best of our knowledge, cytogenetic analyses using FISH with species-specific probes have not been performed in fish hybrids.

Here, we examined the presence or absence of regular pairing of homologous and/or homoeologous chromosomes in testicular cells of sterile clone-origin triploid and inter-group hybrid males to gain insight into the cytogenetic mechanisms underlying the observed malespecific sterility. We used FISH techniques to distinguish chromosomes derived from group B and to identify nucleolar organizing region (NOR) bearing chromosomes.

\section{Material and Methods}

\section{Group Identification and Ploidy Determination of}

Experimental Fish

Two dojo loaches were used in this study: a clone-origin triploid male and an inter-group hybrid male between groups $\mathrm{A}$ and $\mathrm{B}$ wild-type loach. Group A wild type, group B wild type, clone- 
origin triploid, and inter-group hybrid were distinguished by mitochondrial DNA control region haplotype [Morishima et al., 2008a], nuclear DNA-RAG1 gene sequence [Yamada et al., 2015] and RFLP [Fujimoto et al., 2017], and electrophoretic pattern of repetitive sequences ManDra and ManBgl [Fujimoto et al., 2017]. Sample ploidy was determined by flow cytometry as described previously [Morishima et al., 2002]. The triploid loach was caught in Abashiri city, Hokkaido Prefecture. To improve the accuracy of ploidy determination in the triploid, we confirmed the chromosome number in somatic cells using flow cytometry. The inter-group hybrid was induced by artificial fertilization with group B wild-type eggs from Nanae town, Hokkaido Prefecture and group A wild-type sperm from Abashiri city, Hokkaido Prefecture.

\section{Chromosome Preparation}

To prepare chromosome slides from kidneys and testis, goat serum $(100 \mu \mathrm{L} / \mathrm{g}$ body weight) was individually injected 5 and 1 day prior to sacrifice. Subsequently, $0.01 \%$ colchicine in physiological saline $\left(\mathrm{NaCl} 7.5 \mathrm{~g}, \mathrm{KCl} 0.2 \mathrm{~g}, \mathrm{CaCl}_{2} \times 2 \mathrm{H}_{2} \mathrm{O} 0.264 \mathrm{~g}\right.$ $\mathrm{DW} / \mathrm{L}$ ) was injected $2.5 \mathrm{~h}$ prior to sacrifice. After kidney and testis tissues were removed from the body, the tissues were cut into small pieces. These pieces were treated with a hypotonic solution $(0.075 \mathrm{M} \mathrm{KCl})$ for $20 \mathrm{~min}$ and fixed in Carnoy's solution (3:1 methanol/acetic acid) until use. Cell suspensions from the tissues were dropped onto glass slides and air dried. Before being used for FISH, the slides were incubated at $65^{\circ} \mathrm{C}$ for $24 \mathrm{~h}$ for hardening.

\section{Two-Color FISH}

Repetitive sequences (approximately $130 \mathrm{bp}$ ), designated as ManDra, were isolated from group B genomic DNA by DraI restriction enzyme digestion described in Fujimoto et al. [2017]. Plasmids containing inserted ManDra repetitive sequences were used as ManDra probes to distinguish group B-derived chromosomes [Kuroda et al., 2018]. The ManDra probe was labeled with biotin-16-dUTP by Biotin-Nick Translation Mix (Roche).

Human $5.8 \mathrm{~S}+28 \mathrm{~S}$ rDNA sequences were used as probes [Fujiwara et al., 1998] to determine the largest (first) metacentric homologous chromosomes in the dojo loach [Li et al., 2010, 2011, 2012, 2015, 2016; Kuroda et al., 2018]. Two rDNA signals can be detected in diploid loaches and 3 rDNA signals can be detected in triploid loaches. The rDNA probe was labeled with digoxigenin11-dUTP by Dig-Nick Translation Mix (Roche).

Two-color FISH was performed according to Kuroda et al. [2018]. The biotin-labeled ManDra probe was detected with streptavidin, Alexa Fluor 488 conjugate (Thermo Fisher Scientific). The signals were amplified using a biotinylated anti-avidin antibody (Vector Laboratories). The digoxigenin-labeled 5.8S + 28S rDNA probe was detected with anti-digoxigenin-rhodamine, Fab fragments (Roche). The slide was counterstained with 4', 6-diamidino2-phenylindole, dihydrochloride (DAPI).

\section{Statistics}

Data are shown as mean \pm standard deviation (SD). Statistical significance in the number of bivalent chromosomes (II-AA, II$\mathrm{AB}$ ) was confirmed using Student's $t$-test. $p$-values $<0.05$ were considered statistically significant.
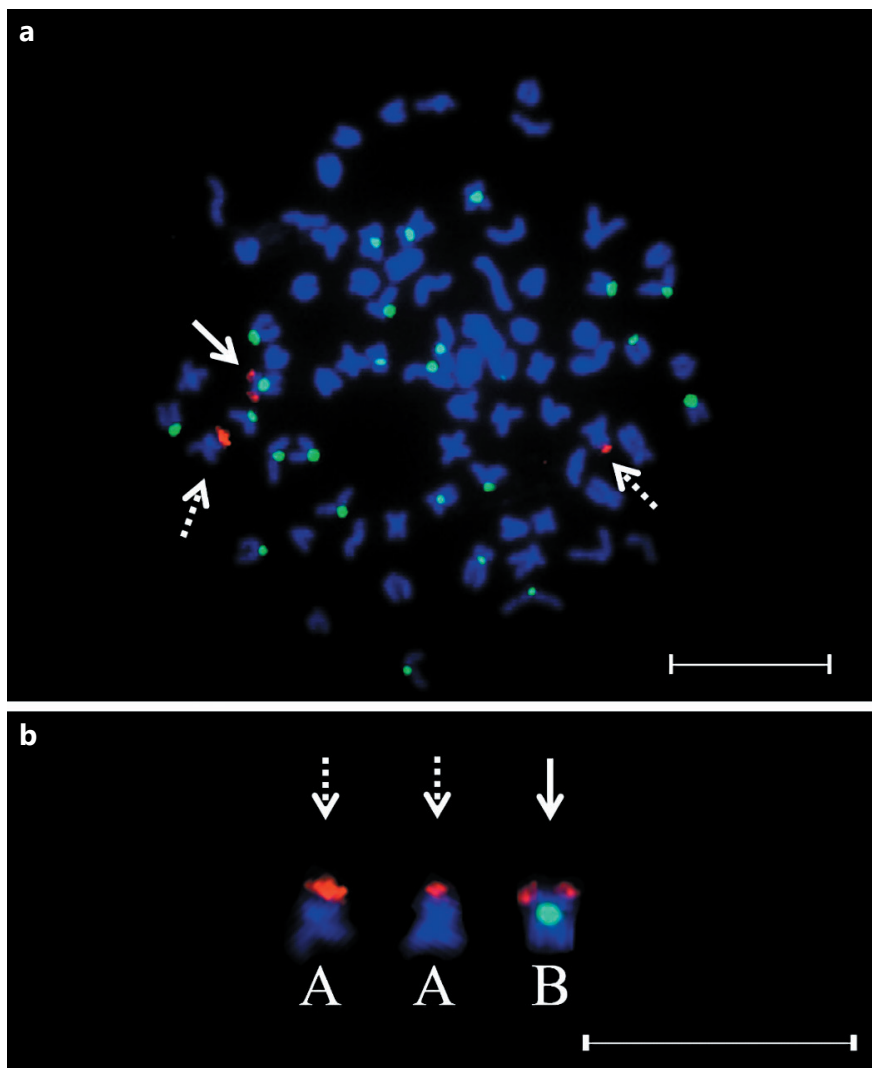

Fig. 1. Two-color FISH using ManDra and $5.8 \mathrm{~S}+28 \mathrm{~S}$ rDNA probes in the somatic cells of the clone-origin triploid male. Representative somatic metaphase (a) and the partial karyotype of the largest homologous chromosomes (b) in the clone-origin triploid after two-color FISH with ManDra and $5.8 \mathrm{~S}+28 \mathrm{~S}$ rDNA probes are shown. The arrow indicates the largest homologous metacentric chromosome with ManDra and rDNA signals. Dotted arrows indicate the largest homologous metacentric chromosomes with rDNA signal but no ManDra signal. The ManDra probe was labeled with biotin-16-dUTP and detected using streptavidin Alexa Fluor 488 conjugate (green). The $5.8 \mathrm{~S}+28 \mathrm{~S}$ rDNA probe was labeled with digoxigenin-11-dUTP and detected using anti-digoxigenin-rhodamine, Fab fragments (red). All chromosomes were counterstained with DAPI (blue). Scale bars, $10 \mu \mathrm{m}$.

\section{Results}

\section{Genomic Composition Analysis in Clone-Origin}

Triploid Male using Two-Color FISH

In a clone-origin triploid sample, somatic metaphases are comprised of 75 chromosomes: 4 out of 5 metaphases showed eutriploidy with 75 chromosomes, while one was from a broken cell with 68 chromosomes. ManDra signals were detected at the centromeric regions of 25 out of 75 chromosomes (Fig. 1a). Therefore, these 25 chromosomes were derived from group $\mathrm{B}$, and the other 50 chro- 
mosomes were derived from group A. Also, $5.8 \mathrm{~S}+28 \mathrm{~S}$ rDNA signals were clearly observed in NORs at the short arms of the 3 largest (first) metacentric homologous chromosomes (Fig. 1, arrow and dotted arrows). ManDra signal was detected in 1 of the 3 rDNA bearing homologous chromosomes (Fig. 1b). Therefore, the genomic composition of the triploid was cytogenetically confirmed to be $\mathrm{AAB}$, with $\mathrm{AB}$ (egg-derived) and $\mathrm{A}$ (sperm-derived) genomes.

\section{Meiosis in Clone-Origin Triploid Male}

Meiotic metaphases of clone-origin triploid males frequently comprised both bivalent and univalent chromosomes, but trivalent chromosomes were scarce. There were 2 types of NOR-bearing chromosomes with rDNA signals among the bivalent chromosomes: bivalent chromosomes paired between homologues from group A, without ManDra signal (II-AA, Fig. 2a, b), and bivalent chromosomes paired between a chromosome without ManDra signal from group A and a chromosome with ManDra signal from group B (II-AB, Fig. 2c, d). Failure of pairing generated 1 ManDra-positive univalent chromosome (I-B) and 2 ManDra-negative univalent chromosomes (I-A, Fig. 2e, f). Infrequent trivalent chromosomes (III-AAB) showed 1 ManDra-positive and 2 ManDra-negative elements among the 3 elements with rDNA signals (Fig. 2g, h).

Using the presence or absence of ManDra signals as the defining criteria, bivalent chromosomes were categorized as II-AA (pairing between chromosomes from group A) and II-AB (pairing between chromosomes from the 2 groups $A$ and $B$ ) (Fig. 2). Univalent chromosomes could also be categorized as I-A and I-B and trivalent chromosomes as III-AAB (Fig. 2). The numbers of uni-, bi-, and trivalent chromosomes largely varied from metaphase to metaphase, but the chromosome number at each metaphase was consistently 75 , equivalent to triploidy (Table 1). Homogenomic bivalent chromosomes (II-AA) were predominant in triploid metaphases, but their number varied between 10 (20 chromosomes) and 24 (48 chromosomes), while the number of heterogenomic bivalent chromosomes (II-AB) varied between 1 ( 2 chromosomes) and 9 (18 chromosomes) (Table 1). Bivalent chromosome number per cell was mean $17.4 \pm 3.0 \mathrm{SD}$ for II-AA, and was significantly larger $(t=19.1, p=1.96 \times$ $\left.10^{-19}<0.05\right)$ than the $3.8 \pm 1.9$ observed for II-AB (Table 1). Accordingly, the mean number of univalent chromosomes per cell was $21.2 \pm 1.8$ and $11.3 \pm 5.4$ for I-B, and I-A, respectively (Table 1). Formation of a trivalent chromosome was difficult, even in a triploid metaphase,
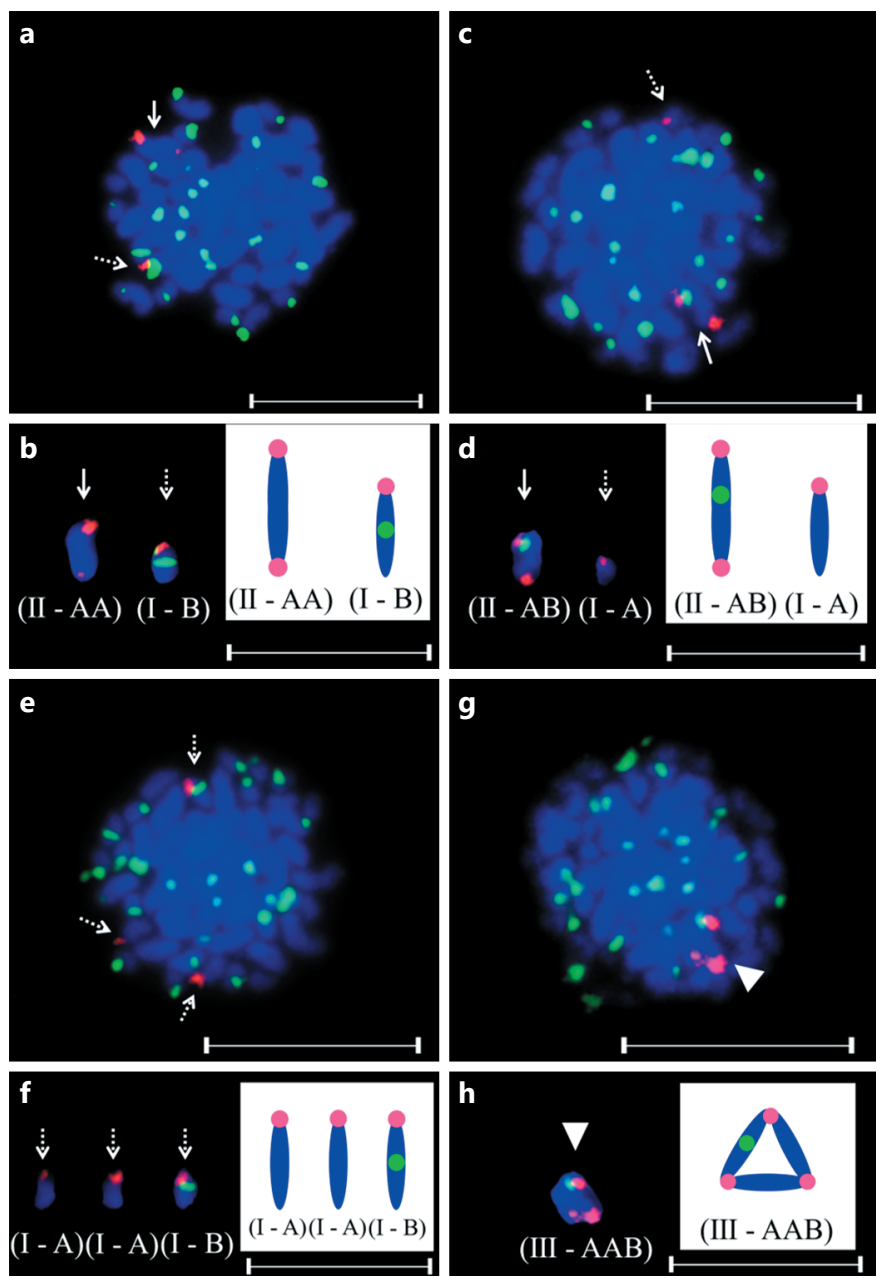

Fig. 2. Two-color FISH with ManDra and $5.8 \mathrm{~S}+28 \mathrm{~S}$ rDNA probes in spermatocytes of clone-origin triploid male. a, c, e, $\mathbf{g}$ Representative meiotic metaphases. b, d, f, h Partial karyotypes and schematic diagrams. Bivalent chromosome that originated from group A and a univalent chromosome that originated from group B (b), bivalent chromosome originating from groups $\mathrm{A}$ and $\mathrm{B}$ and a univalent chromosome that originated from group A (d), three univalent chromosomes that originated from groups A and B (f), and one trivalent chromosome that originated from groups $\mathrm{A}$ and $\mathrm{B}$ (h) in spermatocytes of a clone-origin triploid after two-color FISH with ManDra and 5.8S + 28S rDNA probes are shown. Arrows indicate the bivalent chromosome with 2 rDNA signals. Dotted arrows indicate the univalent chromosomes. The arrowhead indicates the trivalent chromosome with 3 rDNA signals $(\mathbf{g}, \mathbf{h})$. The ManDra probe was labeled with biotin-16-dUTP and detected using streptavidin Alexa Fluor 488 conjugate (green). The $5.8 \mathrm{~S}+$ $28 \mathrm{~S}$ rDNA probe was labeled with digoxigenin-11-dUTP and detected using anti-digoxigenin-rhodamine, Fab fragments (red). All chromosomes were counterstained with DAPI (blue). Scale bars, $10 \mu \mathrm{m}$. 
Table 1. Number of univalent, bivalent, and trivalent chromosomes in meiotic metaphases of clone-origin triploid male

\begin{tabular}{|c|c|c|c|c|c|c|}
\hline \multicolumn{2}{|c|}{ Univalent (I) } & \multicolumn{2}{|c|}{ Bivalent (II) } & \multirow{2}{*}{$\begin{array}{l}\text { Trivalent } \\
\text { (III) } \\
\mathrm{AAB}\end{array}$} & \multirow{2}{*}{$\begin{array}{l}\text { Chromo- } \\
\text { somes }\end{array}$} & \multirow{2}{*}{ Cells } \\
\hline A & B & AA & $\mathrm{AB}$ & & & \\
\hline 1 & 24 & 24 & 1 & 0 & 75 & 1 \\
\hline 2 & 21 & 22 & 4 & 0 & 75 & 1 \\
\hline 5 & 24 & 22 & 1 & 0 & 75 & 1 \\
\hline 4 & 19 & 20 & 6 & 0 & 75 & 2 \\
\hline 5 & 20 & 20 & 5 & 0 & 75 & 1 \\
\hline 9 & 24 & 20 & 1 & 0 & 75 & 1 \\
\hline 6 & 21 & 20 & 4 & 0 & 75 & 1 \\
\hline 8 & 21 & 19 & 4 & 0 & 75 & 1 \\
\hline 9 & 22 & 19 & 3 & 0 & 75 & 2 \\
\hline 10 & 23 & 19 & 2 & 0 & 75 & 1 \\
\hline 9 & 20 & 18 & 5 & 0 & 75 & 3 \\
\hline 11 & 22 & 18 & 3 & 0 & 75 & 3 \\
\hline 10 & 19 & 17 & 6 & 0 & 75 & 1 \\
\hline 11 & 20 & 17 & 5 & 0 & 75 & 1 \\
\hline 13 & 22 & 17 & 3 & 0 & 75 & 2 \\
\hline 12 & 22 & 17 & 2 & 1 & 75 & 1 \\
\hline 14 & 21 & 16 & 4 & 0 & 75 & 1 \\
\hline 16 & 23 & 16 & 2 & 0 & 75 & 3 \\
\hline 17 & 22 & 15 & 3 & 0 & 75 & 1 \\
\hline 17 & 20 & 14 & 5 & 0 & 75 & 1 \\
\hline 18 & 21 & 14 & 4 & 0 & 75 & 1 \\
\hline 20 & 23 & 14 & 2 & 0 & 75 & 1 \\
\hline 17 & 16 & 12 & 9 & 0 & 75 & 1 \\
\hline 20 & 19 & 12 & 6 & 0 & 75 & 1 \\
\hline 23 & 18 & 10 & 7 & 0 & 75 & 1 \\
\hline $\begin{array}{l}11.3 \pm \\
5.4\end{array}$ & $\begin{array}{l}21.2 \pm \\
1.8\end{array}$ & $\begin{array}{l}17.4 \pm \\
3.0\end{array}$ & $\begin{array}{l}3.8 \pm \\
1.9\end{array}$ & & & $\begin{array}{l}\text { Total } \\
34 \\
\text { cells }\end{array}$ \\
\hline
\end{tabular}

The last line shows the mean and standard deviation.

and only 1 cell with trivalent chromosomes was observed in a total of 34 metaphases (Table 1).

\section{Confirmation of Genome Origin in the Hybrid Loach}

Twenty-five ManDra signals were detected in the inter-group hybrid. Therefore, the hybrid comprised 25 chromosomes derived from group A and 25 chromosomes derived from group B (Fig. 3).

\section{Meiosis in Inter-Group Hybrid Male}

Spermatocytes of the inter-group hybrid contained different numbers of bivalent and univalent chromosomes (Fig. 4; Table 2). Twenty-five ManDra probe signals were detected in all 43 meiotic cells examined. Twocolor FISH indicated the presence of heterogenomic bivalent chromosomes (II-AB) formed by pairing between
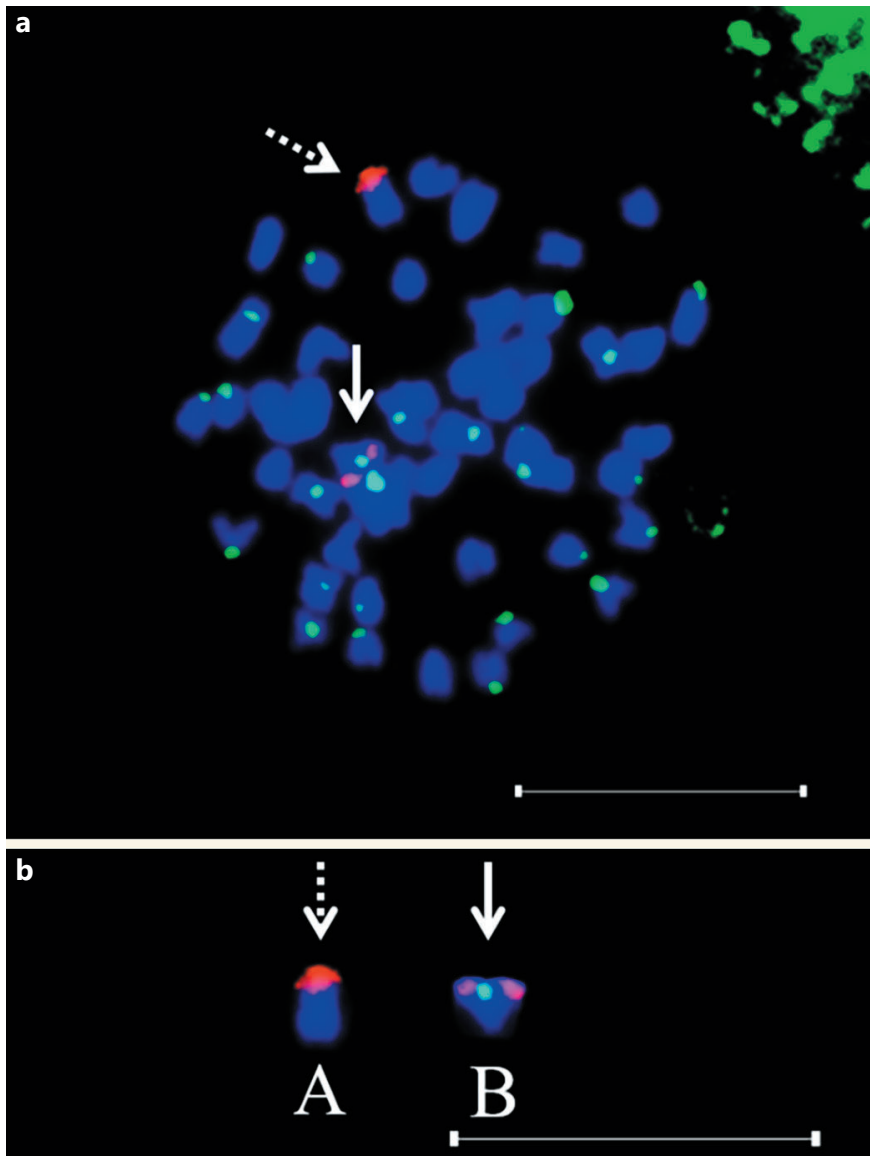

Fig. 3. Two-color FISH with ManDra and 5.8S $+28 \mathrm{~S}$ rDNA probes in somatic cells of the inter-group hybrid. Representative metaphase (a) and partial karyotype of a pair of the largest homologous metacentric chromosomes (b) in somatic cells of hybrid between wild-type loach groups A and B after two-color FISH with ManDra and $5.8 \mathrm{~S}+28 \mathrm{~S}$ rDNA probes are shown. The arrow indicates one of a pair of the largest homologous metacentric chromosomes with ManDra signals. The dotted arrow indicates the other of a pair of the largest homologous metacentric chromosomes without ManDra signals. The ManDra probe was labeled with biotin-16-dUTP and detected using streptavidin Alexa Fluor 488 conjugate (green). A pair of the largest homologous metacentric chromosomes with nucleolar organizing regions (NORs) was determined using the $5.8 \mathrm{~S}+28 \mathrm{~S}$ rDNA probe labeled with digoxigenin-11-dUTP and detected with anti-digoxigenin-rhodamine, Fab fragments (red). All chromosomes were counterstained with DAPI (blue). Scale bars, $10 \mu \mathrm{m}$.

a group A-derived (ManDra-negative) and group B-derived (ManDra-positive) chromosomes in NOR-bearing largest (first) metacentric bivalent chromosomes with rDNA signal (Fig. 4a, b). The mean number of bivalent chromosomes per cell was $21.8 \pm 1.6$ for II-AB (Table 2). The other metaphases showed 2 univalent chromosomes 

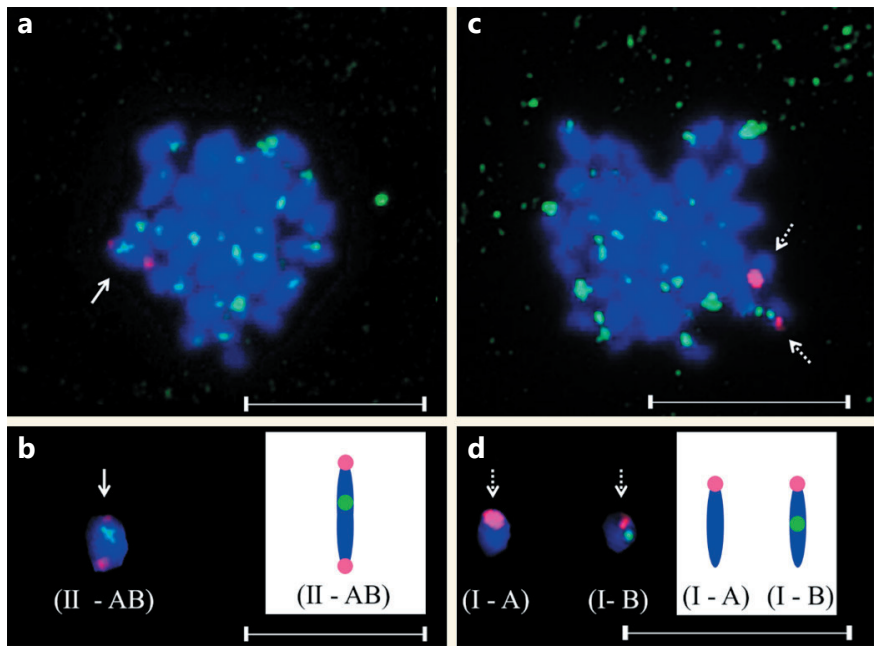

Fig. 4. Two-color FISH with ManDra and 5.8S + 28S rDNA probes in spermatocytes of the inter-group hybrid. Representative meiotic metaphases $(\mathbf{a}, \mathbf{c})$ and partial karyotypes and schematic diagrams (b, d) of the bivalent chromosome (b), and 2 univalent chromosomes (d) in spermatocytes of the inter-group hybrid (between groups A and B) after two-color FISH with ManDra and 5.8S + 28S rDNA probes are shown. The arrow indicates the bivalent chromosome with a ManDra signal and 2 rDNA signals. The dotted arrows indicate the 2 univalent chromosomes with rDNA signals. One of 2 univalent chromosomes with rDNA signals had a ManDra signal. ManDra probe was labeled with biotin-16-dUTP and detected using streptavidin Alexa Fluor 488 conjugate (green). The rDNA sequences of the bivalents were visualized by labeling the $5.8 \mathrm{~S}+28 \mathrm{~S}$ rDNA probe with digoxigenin-11-dUTP and detection using anti-digoxigenin-rhodamine, Fab fragments (red). All chromosomes were counterstained with DAPI (blue). Scale bars, 10 $\mu \mathrm{m}$.

from NOR bearing chromosomes with rDNA signal, one also had the ManDra signal from group B (I-B) and another had no signal from group A (I-A) (Fig. 4c, d). The mean number of univalent chromosomes per cell was 3.2 \pm SD 1.6 for I-A and I-B (Table 2). The frequency of univalent chromosomes was reduced due to the increased number of bivalent chromosomes. Most metaphases (36/43 cells) included 21-23 bivalent chromosomes and 4-8 univalent chromosomes.

\section{Discussion}

In the clone-origin triploid male, bivalent chromosomes preferentially synapsed between chromosomes derived from the same group, group A. Consequently, univalent chromosomes of group A chromosomes (mean $11.3 \pm 5.4$ ) were observed less frequently than those of
Table 2. Number of univalent and bivalent chromosomes in meiotic metaphases of inter-group hybrid male between groups A and $\mathrm{B}$

\begin{tabular}{|c|c|c|c|c|}
\hline \multicolumn{2}{|c|}{ Univalent (I) } & \multirow{2}{*}{$\frac{\text { Bivalent (II) }}{\mathrm{AB}}$} & \multirow{2}{*}{$\begin{array}{l}\text { Chromo- } \\
\text { somes }\end{array}$} & \multirow[t]{2}{*}{ Cells } \\
\hline A & B & & & \\
\hline 8 & 8 & 17 & 50 & 1 \\
\hline 7 & 7 & 18 & 50 & 2 \\
\hline 6 & 6 & 19 & 50 & 1 \\
\hline 5 & 5 & 20 & 50 & 1 \\
\hline 4 & 4 & 21 & 50 & 10 \\
\hline 3 & 3 & 22 & 50 & 11 \\
\hline 2 & 2 & 23 & 50 & 15 \\
\hline 1 & 1 & 24 & 50 & 2 \\
\hline $3.2 \pm 1.6$ & $3.2 \pm 1.6$ & $21.8 \pm 1.6$ & & Total \\
\hline
\end{tabular}

The last line shows the mean and standard deviation.

group B $(21.2 \pm 1.8)$, because they tended to pair with their counterpart from the same origin. The numbers of bivalent and univalent chromosomes largely varied among spermatocytes, and no clear modal configuration was observed. A trivalent chromosome was identified in only one spermatocyte. Taken together, these results show that synapsis occurs between homologous chromosomes derived from the same origin with higher affinity, but that synapsis between chromosomes derived from different origins is also possible, but occurs infrequently. Consequently, in triploid meiosis, 10-24 II-AA, 1-9 II$\mathrm{AB}, 1-23$ I-A, and 16-24 I-B appeared. Bivalent chromosomes should segregate to each pole, while univalent chromosomes might segregate randomly. Therefore, various kinds of aneuploid spermatozoa were predicted in the clone-origin triploid, but no or very few functional sperm were produced. Natural clone-origin triploid males exhibit triploid and hexaploid cell populations in testis, but functional haploid spermatozoa are very rarely observed, and most spermatozoa and spermatozoon-like cells are morphologically abnormal and non-motile [Oshima et al., 2005]. Therefore, in the clone-origin triploid male, replication would proceed to form hexaploid cells with 6C DNA content. These cells may then accumulate in testis without completing the first meiotic division due to their aberrant chromosome configurations including irregular univalent chromosomes and trivalent chromosomes. Spermiogenesis without completion of meiosis was reported in the diploid interspecific medaka hybrid, in which non-motile spermatozoon-like cells with tetraploidy (4C DNA content) were formed [Shimizu et al., 
1997]. A similar phenomenon may occur in clone-origin triploid testis with germ cells being able to replicate but unable to complete regular meiosis, leading to the appearance of abnormal spermatozoon-like cells. This reproductive feature differs greatly from those observed in the clone-origin triploid female [Morishima et al., 2008b]. In the triploid female, 25 bivalent chromosomes were observed in germinal vesicles of mature oocytes, and fertile haploid eggs were produced, presumably by eliminating unmatched genomes and subsequent quasi-normal bivalent chromosome formation during meiosis (meiotic hybridogenesis) [Morishima et al., 2008b]. Here, meiotic cells composed of 25 bivalent chromosomes, without any univalent and trivalent chromosomes, were never observed, suggesting that genome elimination occurs in later stages or that there is no genome elimination at all.

The inter-group hybrid male, between groups A and $\mathrm{B}$, showed post-zygotic sterility because it produced non-motile spermatozoa or spermatozoon cells [AriasRodriguez et al., 2010]. Here, we observed that most chromosomes (34-48 of 50) paired to form bivalent chromosomes (17-24 of 25) between heterogenomic chromosomes derived from groups A and B, but 2-16 chromosomes, which could not find a counterpart with which to pair, formed 2-16 univalent chromosomes in the hybrid male. Although univalent chromosomes were observed in all meiotic cells, the number of bivalent and univalent chromosomes differed in each cell. As observed in other sterile interspecific hybrids of vertebrates [Müller, 1977; Shimizu et al., 1997; Bhattacharyya et al., 2013; Islam et al., 2013; Torgasheva et al., 2016], failure of synapsis was observed in the inter-group hybrid loach male. Coexistence of bivalent and univalent chromosomes may cause the formation of aneuploid gametes due to random univalent segregation, but hybrid males produced haploid, diploid, and tetraploid spermatozoa or spermatozoon-like cells with low ability to generate next generation zygotes [Arias-Rodriguez et al., 2010].

Similar to the triploid males, tetraploid cells with a $4 \mathrm{C}$ content were observed in the sperm and testis of intergroup hybrid males, and they were considered to be spermatocytes that underwent replication but could not complete meiosis [Arias-Rodriguez et al., 2010]. Thus, tetraploid spermatocytes presumably accumulated in the testes and differentiated into spermatozoon-like cells without completion of meiosis. Such a situation was reported in interspecific medaka hybrids [Shimizu et al., 1997].

In a clonal diploid loach, unreduced diploid gametes are produced by pairing between sister chromosomes doubled from each ancestral chromosome before meiosis (premeiotic endomitosis) [Kuroda et al., 2018]. If the same mechanism acted in clone-origin triploid males and inter-group hybrid males, 50 ManDra signals should be detected. In this study, however, 25 ManDra signals were detected in all meiotic cells observed, suggesting no occurrence of premeiotic endomitosis. It is unclear why premeiotic endomitosis did not occur in the inter-group hybrid male $(\mathrm{AB})$, which had a genomic constitution similar to the clonal male $(\mathrm{AB})$.

Analysis of mitochondrial DNA-control regions indicated that the genetic distance between the 2 groups, A and $\mathrm{B}$, is larger than that between different species, $M$. mizolepis and M. fossilis [Morishima et al., 2008a]. Similar results were obtained by analyses of allozyme variation and the nuclear DNA-RAG1 gene [Khan and Arai, 2000; Yamada et al., 2015]. The presence of asynapsis observed in our study provides cytogenetic evidence that distant genetic differentiation occurred between the 2 groups of dojo loach.

The reproductive system in hybrids of European spined loach (genus Cobitis) is similar to dojo loach. The spined loach hybrid complex is composed of di-, tri-, and tetraploid [Janko et al., 2007a]. Moreover, clonal lineages originating from hybridization between different Cobitis species are present [Janko et al., 2007a]. Clonally reproducing diploid females are present in both the dojo and spined loach [Janko et al., 2007a, b; Choleva et al., 2012]. However, in triploid females, the clone-origin triploid dojo loach produces haploid eggs by a mechanism of meiotic hybridogenesis [Morishima et al., 2008b], but the triploid spined loach lays unreduced triploid eggs which develop by gynogenesis [Janko et al., 2007b]. On the other hand, the males showed common characteristics. Spermatozoa from a triploid male of spined loach did not move after adding ambient water [Vasil'ev et al., 2003]. Moreover, diploid hybrid males were sterile [Choleva et al., 2012; Juchno and Boron, 2018]. Thus, sterility of the male spined loach may be caused by aberrant meiotic configurations, as observed in dojo loach.

Our results show that asynapsis and irregular synapsis occurred in triploid and hybrid males containing 2 different genomes and that this may lead to the formation of sterile spermatozoa. These results will help us to understand hybrid sterility from the viewpoint of synapsis behavior.
Kuroda/Fujimoto/Murakami/Yamaha/ Arai 


\section{Acknowledgements}

We thank Dr. Atsushi Fujiwara (National Research Institute of Fisheries Science, Japan Fisheries Research and Education Agency) for technical advice. This study was supported by Grantsin-Aid from JSPS (Japan Society for the Promotion of Science) KAKENHI Grant Number 15H02457, Young Scientists (A) Grant Number 25712021, and JSPS Research Fellow Number 17J01971.

\section{Statement of Ethics}

This study was performed according to the Guide for the Care and Use of Laboratory Animals in Hokkaido University. All animal experiments were approved by the animal study ethical committee of Hokkaido University (Approval number 29-3).

\section{Disclosure Statement}

The authors have no conflicts of interest to declare.

\section{References}

Arai K, Fujimoto T: Genomic constitution and atypical reproduction in polyploid and unisexual lineages of the Misgurnus loach, a teleost fish. Cytogenet Genome Res 140:226-240 (2013).

-Arias-Rodriguez L, Morishima K, Arai K: Genetically diversified populations in the loach Misgurnus anguillicaudatus inferred from newly developed microsatellite markers. Mol Ecol Notes 7:82-85 (2007).

-Arias-Rodriguez L, Yasui GS, Arai K: Disruption of normal meiosis in artificial inter-populational hybrid females of Misgurnus loach. Genetica 136:49-56 (2009).

Arias-Rodriguez L, Yasui GS, Kusuda S, Arai K: Reproductive and genetic capacity of spermatozoa of inter-populational hybrid males in the loach, Misgurnus anguillicaudatus. J Appl Ichthyol 26:653-658 (2010).

Bhattacharyya T, Gregorova S, Mihola O, Anger M, Sebestova J, et al: Mechanistic basis of infertility of mouse intersubspecific hybrids. Proc Natl Acad Sci USA 110:E468-E477 (2013).

- Choleva L, Janko K, De Gelas K, Bohlen J, Šlechtová V, et al: Synthesis of clonality and polyploidy in vertebrate animals by hybridization between two sexual species. Evolution 66:2191-2203 (2012).

Dawley RM: An introduction to unisexual vertebrates, in Dawley RM, Bogart JP (eds): Evolution and Ecology of Unisexual Vertebrates, pp 1-18 (New York State Museum, Albany 1989).

- Fujimoto T, Yamada A, Kodo Y, Nakaya K, Okubo-Murata M, et al: Development of nuclear DNA markers to characterize genetically diverse groups of Misgurnus anguillicaudatus and its closely related species. Fish Sci 83:743756 (2017)

Fujiwara A, Abe S, Yamaha E, Yamazaki F, Yoshida CM: Chromosomal localization and heterochromatin association of ribosomal RNA gene loci and silver-stained nucleolar organizer regions in salmonid fishes. Chromosome Res 6:463-471 (1998).
Islam FB, Ishishita S, Uno Y, Mollah MBR, Srikulnath K, Matsuda Y: Male hybrid sterility in the mule duck is associated with meiotic arrest in primary spermatocytes. J Poultry Sci 50:311-320 (2013).

Itono M, Morishima K, Fujimoto T, Bando E, Yamaha E, Arai K: Premeiotic endomitosis produces diploid eggs in the natural clone loach, Misgurnus anguillicaudatus (Teleostei: Cobitidae). J Exp Zool A Comp Exp Biol 305:513523 (2006).

Itono M, Okabayashi N, Morishima K, Fujimoto $\mathrm{T}$, Yoshikawa H, et al: Cytological mechanisms of gynogenesis and sperm incorporation in unreduced diploid eggs of the clonal loach, Misgurnus anguillicaudatus (Teleostei: Cobitidae). J Exp Zool A Comp Exp Biol 307: 35-50 (2007).

Janko K, Flajšhans M, Choleva L, Bohlen J, Slechtova V, et al: Diversity of European spined loaches (genus Cobitis L.): an update of the geographic distribution of the Cobitis taenia hybrid complex with a description of new molecular tools for species and hybrid determination. J. Fish Biol 71:387-408 (2007a).

Janko K, Bohlen J, Lamatsch D, Flajšhans M, Epplen JT, et al: The gynogenetic reproduction of diploid and triploid hybrid spined loaches (Cobitis: Teleostei), and their ability to establish successful clonal lineages-on the evolution of polyploidy in asexual vertebrates. Genetica 131:185-194 (2007b).

Juchno D, Boroń A: Histological evidence that diploid hybrids of Cobitis taenia and C. elongatoides (Teleostei, Cobitidae) develop into fertile females and sterile males. Hydrobiologia 814:147-159 (2018).

Khan MR, Arai K: Allozyme variation and genetic differentiation in the loach Misgurnus anguillicaudatus. Fish Sci 66:211-222 (2000).

Kuroda M, Fujimoto T, Murakami M, Yamaha E, Arai K: Clonal reproduction assured by sister chromosome pairing in dojo loach, a teleost fish. Chromosome Res 26:243-253 (2018).

Li YJ, Tian Y, Zhang MZ, Tian PP, Yu Z, et al: Chromosome banding and FISH with rDNA probe in the diploid and tetraploid loach Misgurnus anguillicaudatus. Ichthyol Res 57: 358-366 (2010).
Li YJ, Yu Z, Zhang MZ, Qian C, Abe S, Arai K: The origin of natural tetraploid loach Misgurnus anguillicaudatus (Teleostei: Cobitidae) inferred from meiotic chromosome configurations. Genetica 139:805-811 (2011).

Li YJ, Yu Z, Zhang MZ, Qian C, Abe S, Arai K: Induction of viable gynogenetic progeny using eggs and UV-irradiated sperm from the Chinese tetraploid loach, Misgurnus anguillicaudatus. Aquac Int 21:759-768 (2012).

- Li YJ, Gao YC, Zhou H, Ma HY, Li JQ, Arai K: Meiotic chromosome configurations in triploid progeny from reciprocal crosses between wild-type diploid and natural tetraploid loach Misgurnus anguillicaudatus in China. Genetica 143:555-562 (2015).

Li YJ, Gao YC, Zhou H, Ma HY, Lin ZQ, et al: Aneuploid progenies of triploid hybrids between diploid and tetraploid loach Misgurnus anguillicaudatus in China. Genetica 144:601609 (2016).

Lutes AA, Neaves WB, Baumann DP, Wiegraebe W, Baumann P: Sister chromosome pairing maintains heterozygosity in parthenogenetic lizards. Nature 464:283-286 (2010).

Morishima K, Horie S, Yamaha E, Arai K: A cryptic clonal line of the loach Misgurnus anguillicaudatus (Teleostei: Cobitidae) evidenced by induced gynogenesis, interspecific hybridization, microsatellite genotyping and multilocus DNA fingerprinting. Zoolog Sci 19: 565-575 (2002).

-Morishima K, Nakamura-Shiokawa Y, Bando E, Li YJ, Boroń A, et al: Cryptic clonal lineages and genetic diversity in the loach Misgurnus anguillicaudatus (Teleostei: Cobitidae) inferred from nuclear and mitochondrial DNA analyses. Genetica 132:159-171 (2008a).

Morishima K, Yoshikawa H, Arai K: Meiotic hybridogenesis in triploid Misgurnus loach derived from a clonal lineage. Heredity 100: 581-586 (2008b).

Müller WP: Diplotene chromosomes of Xenopus hybrid oocytes. Chromosoma 59:273 (1977).

Oshima K, Morishima K, Yamaha E, Arai K: Reproductive capacity of triploid loaches obtained from Hokkaido Island, Japan. Ichthyol Res 52:1-8 (2005). 
-Schwenk K, Brede N, Streit B: Extent, processes and evolutionary impact of interspecific hybridization in animals. Philos Trans R Soc Lond B Biol Sci 363:2805-2811 (2008).

-Shimizu Y, Shibata N, Yamashita M: Spermiogenesis without preceding meiosis in the hybrid medaka between Oryzias latipes and O. curvinotus. J Exp Zool 279:102-112 (1997).

-Suzuki R, Oshiro T, Nakanishi T: Survival, growth and fertility of gynogenetic diploids induced in the cyprinid loach, Misgurnus anguillicaudatus. Aquaculture 48:45-55 (1985).

Torgasheva AA, Borodin PM: Cytological basis of sterility in male and female hybrids between sibling species of grey voles Microtus arvalis and M. levis. Sci Rep 6:36564 (2016).

Vasil'ev VP, Akimova NV, Emel'yanova NG, Pavlov DA, Vasil'eva ED: Reproductive capacities in the polyploid males of spined loaches from the unisexual-bisexual complex, occurred in the Moscow River. Folia Biologica 51:67-73 (2003).

Vrijenhoek RC: Unisexual fish: model systems for studying ecology and evolution. Annu Rev Ecol Syst 25:71-96 (1994).

- Yamada A, Kodo Y, Murakami M, Kuroda M, Aoki T, et al: Hybrid origin of gynogenetic clones and the introgression of their mito- chondrial genome into sexual diploids through meiotic hybridogenesis in the loach, Misgurnus anguillicuadatus. J Exp Zool A Ecol Genet Physiol 323:593-606 (2015).

Yoshikawa H, Morishima K, Kusuda S, Yamaha E, Arai K: Diploid sperm produced by artificially sex-reversed clone loaches. J Exp Zool A Ecol Genet Physiol 307:75-83 (2007).

Yoshikawa H, Morishima K, Fujimoto T, Asito T, Kobayashi T, et al: Chromosome doubling in early spermatogonia produces diploid spermatozoa in a natural clonal fish. Biol Reprod 80:973-979 (2009). 